\title{
Direct determination of III-V semiconductor surface band gaps
}

\author{
H. Carstensen, R. Claessen, R. Manzke, and M. Skibowski \\ Institut für Experimentalphysik, Universität Kiel, Olshausenstrasse 40, D-2300 Kiel 1, Federal Republic of Germany
}

(Received 8 December 1989)

\begin{abstract}
Applying in situ combination of angle-resolved photoemission and inverse photoemission to cleaved III-V compound semiconductor (110) surfaces, we have determined the surface band gaps between the filled anion-derived dangling-bound state $A_{5}$ and the empty cation-derived state $C_{3}$ at the $\bar{\Gamma}, \bar{X}^{\prime}, \bar{X}$, and $\bar{M}$ points of the surface Brillouin zone. The values of the surface band gaps of the six compounds under study are found to increase in this sequence and seem to be correlated with the fundamental bulk energy gaps. The results are compared with optical surface excitations as well as electron-energy-loss experiments. The low-lying transition energies agree well with our surface band gaps at the $\bar{X}^{\prime}$ and $\bar{X}$ points.
\end{abstract}

\section{INTRODUCTION}

Angle-resolved photoemission ${ }^{1}$ (ARPES) and inversephotoemission $^{2}$ (ARIPES) spectroscopies are efficient tools for studying the electronic band structure of occupied and unoccupied states. Both methods provide information about energy and momentum simultaneously. Photoemission probes the electronic states below the Fermi level $E_{F}$ and above the vacuum level $E_{\text {vac }}$. The energy range between $E_{F}$ and $E_{\mathrm{vac}}$ is accessible by the recently developed technique of inverse photoemission. Applying ARPES or ARIPES, several groups have investigated the electronic structure of cleaved (110) surfaces of several III-V semiconductor compounds. ${ }^{1,3-7}$ We have combined both methods in situ on a common energy scale to obtain new information about the surface states close to the Fermi level.

On the other hand, surface-sensitive optical ${ }^{8,9}$ and electron-energy-loss spectroscopy ${ }^{10,11}$ (EELS) have been developed in the last few years. Optical transitions between filled and empty states of (110) surfaces of III-V compound semiconductors have been detected by measuring the differential surface reflectivity (DSR) of a clean and a contaminated surface ${ }^{8,12,13}$ or by analyzing the polarization-modulated reflectivity (PMR). ${ }^{9,14}$ For GaAs(110) (Refs. 10 and 11) and GaP(110) (Ref. 10), surface-electronic excitations have been studied by the azimuthal dependence of high-resolution EELS spectra. These measurements determine the energy difference between filled and empty surface states, but, in general, do not resolve the momentum.

Although the (110) surface of III-V compound semiconductors is not that commonly used in technology, most theoretical and experimental work has been carried out on this surface. It is the only nonpolar surface and can easily be prepared as it is the natural cleavage plane. The creation of an ideal (110) surface leaves two dangling bonds per unit cell directed out of the surface associated with two electronic surface bands inside the fundamental band gap. ${ }^{15}$ Since the early work of MacRae and Gobeli, ${ }^{16}$ it is known that this bulklike surface is energeti- cally unstable: The group-V atoms move out of the surface plane and the group-III atoms move towards the bulk. The displacement of the topmost atoms can be described by a relaxation angle $\omega$ of about $30^{\circ}$ for all $A^{\mathrm{III}} B^{\mathrm{V}}(110)$ surfaces. ${ }^{17}$ This relaxation of the surface atoms is intimately connected with an electronic rearrangement forming an empty dangling bond (DB) at the surface cation and a doubly occupied DB at the surface anion. ${ }^{18}$ The associated dispersive surface bands $C_{3}$ and $A_{5}$ are calculated to be pushed out of the fundamental bulk gap with increasing relaxation. ${ }^{15,19,20}$ The aim of the present study is to measure the energy of the filled DB state by ARPES and of the unoccupied DB state by ARIPES in situ for several III-V compounds and combine the results on a common energy scale in order to determine the surface band gaps at all high-symmetry points $\left(\bar{\Gamma}, \bar{X}^{\prime}, \bar{X}\right.$, and $\left.\bar{M}\right)$ of the surface Brillouin zone (SBZ).

After a brief description of the apparatus, the sample preparation, and energy calibration, we present as an example the results for the surface band gaps for $\mathrm{GaSb}(110)$ at different points of the SBZ and, in order to study the chemical trends, the results for six different III-V semiconductor compounds at the $\bar{X}^{\prime}$ point. The results are compared with surface-band-structure calculations and optical and electron-energy-loss data.

\section{EXPERIMENTAL}

The experiments were performed using an ultrahighvacuum (UHV) system consisting of a cleavage chamber, a low-energy electron-diffraction (LEED) and preparation chamber, and the spectroscopy chamber, all coupled by magnetically driven transfer rods. The spectroscopy chamber (Fig. 1) contains a low-energy-electron source (denoted 1 in Fig. 1) and a bandpass photon detector (2) for ARIPES, and a rare-gas discharge lamp (3) and a modified cylindrical mirror analyzer (CMA) (4) for ARPES. $^{21}$ All components are arranged in the same plane (the bandpass detector can be moved out of the line of sight of the lamp), and the angle between the photon 


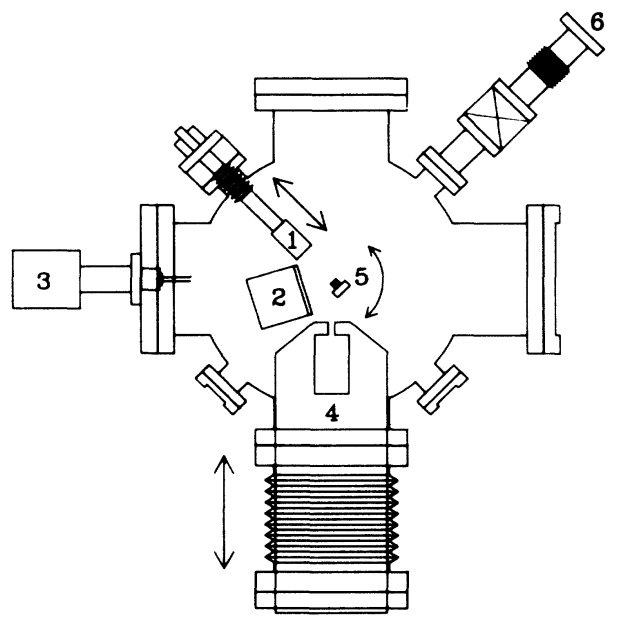

FIG. 1. Experimental setup for combined angle-resolved photoemission and inverse-photoemission spectroscopy. The components are (1) low-energy electron source, (2) bandpass photon detector, (3) rare-gas discharge lamp, (4) modified cylindrical mirror analyzer, (5) sample, and (6) transfer to sample input and preparation chamber.

and electron beam direction is about $45^{\circ}$ for both spectroscopies. The electron source has an energy resolution of $220 \mathrm{meV}$ [full width at half maximum (FWHM)] and a wave-vector resolution of $\approx 0.08 \AA^{-1}$. The electroninduced bremsstrahlung is detected by $h v_{\mathrm{IPE}}=9.9 \mathrm{eV}$ (IPE denotes inverse photoemission) by an efficient bandpass detector consisting of an open $\mathrm{Cu}-\mathrm{Be}$ multiplier

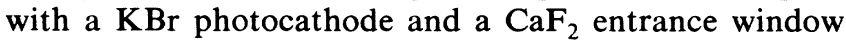
$(\Delta E=600 \mathrm{meV}) .{ }^{22}$ Photoelectrons are excited by He I radiation $\left(h v_{\mathrm{PE}}=21.2 \mathrm{eV}\right)(\mathrm{PE}$ denotes photoemission) and their energy is measured by the CMA $(\Delta E=200 \mathrm{meV})$ modified for angle-resolved measurements by an aperture $\left(\Delta \vartheta= \pm 2^{\circ}\right)$ in front of the CMA.

The samples (GaP, GaAs, GaSb, InP, InAs, and InSb) were $6 \times 8 \times 15-\mathrm{mm}^{3}$ bars of $p$ - or $n$-type single crystals (MCP, London) cleaved by the common anvil and wedge technique with notches parallel to the (110) surface. Clean and mirrorlike sample surfaces were obtained applying this procedure. In order to avoid charging, Ohmic contacts were alloyed (In for $p$-doped and Au-Ge-Ni for $n$-doped samples) under inert-gas atmosphere on one side of the samples and were tested by $I-V$ measurements. Photoemission experiments were performed before and after inverse photoemission to check possible bandbending effects induced by electron-beam irradiation or adsorbates from the electron gun.

In photoemission (PE) or inverse-photoemission (IPE) investigations of metallic samples, an absolute energy scale is usually determined by measuring the energy position of the Fermi edge, $E_{F}$. For semiconductors, however, the relevant reference energy for comparing the results either to band-structure calculations or to other spectroscopies (optical measurements, EELS, etc.) is the valence-band maximum $E_{\mathrm{VBM}}$. The determination of $E_{\mathrm{VBM}}$ with PE (or the conduction-band minimum $E_{\mathrm{CBM}}$ with IPE) is somewhat difficult, since, because of bulk band dispersion perpendicular to the sample surface, variation of the photon energy is required, and the onset of the spectra is not as sharp as a Fermi edge. Furthermore, defects obtained after cleavage or induced by adsorbates may cause irreproducible band bending, so a comparison of PE and IPE results needs the in situ combination of these spectroscopies at the same sample surface.

For the determination of surface band gaps by combined ARPES and ARIPES, we established a common energy scale by measuring the energy of the electrons from the inverse-photoemission electron source with the photoemission-energy analyzer. To illustrate the calibration method, the top of Fig. 2 shows as an example an ARPES (left-hand side) and an ARIPES spectrum (righthand side) of $\mathrm{GaSb}(110)$ with the occupied $\left(A_{5}\right)$ and unoccupied $\left(C_{3}\right)$ DB states located at the $\bar{X}^{\prime}$ point by choosing the appropriate polar and azimuthal electron angles. The primary experimental energy scales for photoemission and inverse photoemission are given by the voltage of the cylindrical mirror analyzer, $U_{\mathrm{CMA}}$, and the acceleration voltage of the electron gun, $U_{\text {gun }}$ (both taken
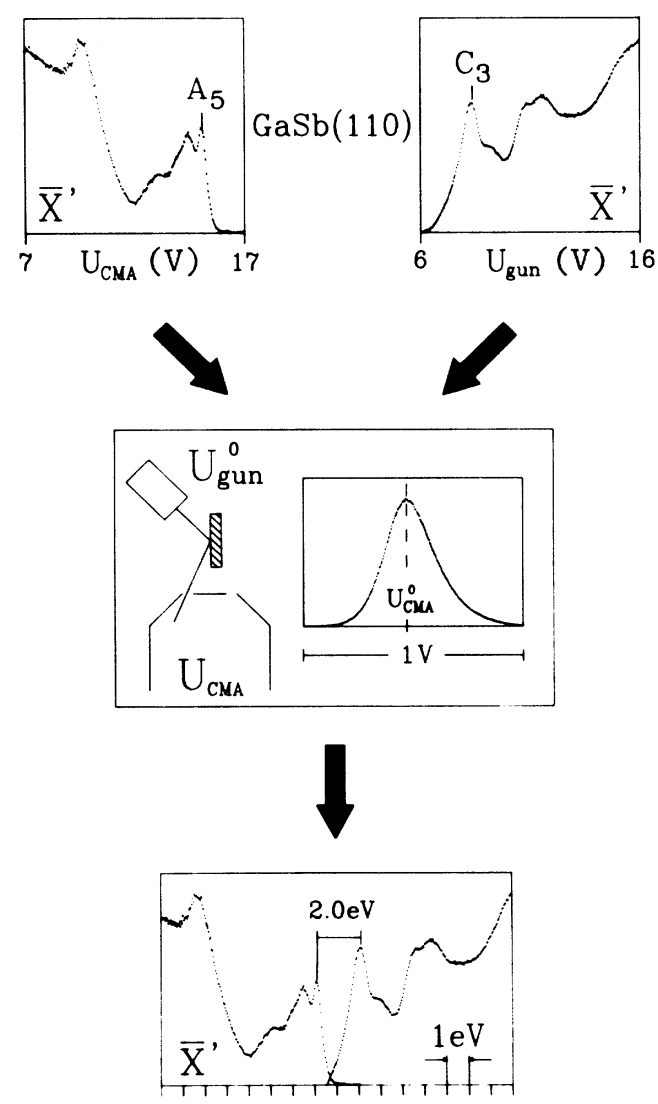

FIG. 2. Determination of a common energy scale for ARPES and ARIPES by measuring the energy distribution of electrons from the inverse-photoemission gun with the electron-energy analyzer (CMA) used for photoemission. Upper part, original experimental spectra; middle part, energy calibration, lower part, combination of experimental spectra on a common energy scale (for further information, see text). 
as positive). The energy difference $E_{\mathrm{IPE}}-E_{\mathrm{PE}}$ between unoccupied and occupied electronic states is determined by energy conservation in IPE and PE:

$E_{\mathrm{IPE}}-E_{\mathrm{PE}}=|e| U_{\mathrm{gun}}-|e| U_{\mathrm{CMA}}+h v_{\mathrm{PE}}-h v_{\mathrm{IPE}}-\Delta E$,

where $h v_{\mathrm{PE}}(21.2 \mathrm{eV})$ and $h v_{\mathrm{IPE}}(9.9 \mathrm{eV})$ are the photon energies used in PE and IPE, and $\Delta E$ is the energy offset associated with the difference in work function of the electron-energy analyzer and the electron gun. The only unknown quantity, $\Delta E$, can simply be determined by reflecting the electron beam (with a given acceleration voltage $U_{\text {gun }}^{0}$ ) at the sample into the CMA and measuring the position of the maximum of the energy distribution $\left(|e| U_{\mathrm{CMA}}^{0}\right)$ for the elastically scattered electrons. This is shown schematically in the middle of Fig. 2. $\Delta E$ is then given by

$$
\Delta E=|e| U_{\text {gun }}^{0}-|e| U_{\mathrm{CMA}}^{0} .
$$

This way a common energy scale is established for both spectroscopies without using any special reference level like $E_{F}$ or $E_{\mathrm{VBM}}$ and a direct determination of the surface band gaps becomes possible. The bottom of Fig. 2 shows the combined ARPES and ARIPES spectra for $\mathrm{GaSb}(110)$. The energy difference between $C_{3}$ and $A_{5}$ at the $\bar{X}^{\prime}$ point can directly be determined as $2.0 \mathrm{eV}$. It should be mentioned here that such a straightforward comparison is only applicable for surface-derived states because they have no dispersion perpendicular to the sample surface.

\section{RESULTS AND DISCUSSION}

In angle-resolved photoemission and inverse photoemission particular points in the surface Brillouin zone can be investigated by the appropriate choice of the azimuthal and polar electron angle, $\varphi$ and $\vartheta$, respectively, together with the kinetic energy $E_{\text {kin }}: \quad\left|k_{\|}\right|$ $=\left(2 m E_{\mathrm{kin}} / \hbar^{2}\right)^{1 / 2} \sin \vartheta$. With the common energy scale as described above, the energy differences of the surfacederived maxima in these spectra can be directly determined. As an example, Fig. 3 shows the results for $\mathrm{GaSb}(110)$ at the high-symmetry points $\bar{\Gamma}, \bar{X}^{\prime}, \bar{X}$, and $\bar{M}$ of the SBZ (see inset). The electron angles in the experimental spectra are chosen so that the dangling-bond surface states $A_{5}$ and $C_{3}$ (marked by bars) are associated with kinetic energies corresponding to the symmetry points indicated. The relatively slow onset of the inverse-photoemission spectra originates from the halfwidth and asymmetry of the spectrometer function of the bandpass detector, which has been measured with synchrotron radiation. Thus, it was possible to deconvolute the ARIPES spectra in order to obtain higher accuracy in the final evaluation, but in this paper all spectra are shown in their original form. The complete $\vartheta$-dependent series of photoemission and inverse-photoemission spectra of GaSb (Ref. 23) show a dispersion of the peaks marked in Fig. 3 which follow the periodicity of the SBZ. Furthermore, a strong sensitivity to adsorbates has been found. These facts and the comparison with theory identifies these structures as being due to the occupiedanion-derived $\left(A_{5}\right)$ and unoccupied-cation-derived $\left(C_{3}\right)$ dangling-bond surface states. As seen in Fig. 3, for $\mathrm{GaSb}(110)$ the emission from the DB states at the $\bar{\Gamma}$ and $\bar{M}$ points is only weak, but for $\bar{X}^{\prime}$ and $\bar{X}$ the peaks become very pronounced. This behavior is observed for all III-V semiconductor compounds under study. It seems that for $\bar{\Gamma}$ and $\bar{M}$ the dangling-bond states are resonant or very close to the projected bulk band region, whereas they become free surface states at $\bar{X}^{\prime}$ and $\bar{X}$. The sequence of surface band gaps increasing from $\bar{\Gamma}$ over $\bar{X}^{\prime}$ and $\bar{X}$ to $\bar{M}$ is the same for all investigated samples with a small but not significant deviation for the phosphorcontaining III-V compound semiconductors. The experimental values for the surface band gaps are collected in Table I, together with their bulk band gaps. ${ }^{24}$ For $\mathrm{GaSb}(110)$ we obtain $1.9 \mathrm{eV}$ at $\bar{\Gamma}, 2.0 \mathrm{eV}$ at $\bar{X}^{\prime}, 2.3 \mathrm{eV}$ at $\bar{X}$ and $2.7 \mathrm{eV}$ at $\bar{M}$, with an accuracy of about $\pm 0.05 \mathrm{eV}$ for $\bar{X}^{\prime}$ and $\bar{X}$ and, due to the weakness of the structures, $\pm 0.15 \mathrm{eV}$ for $\bar{\Gamma}$ and $\bar{M}$. Semiempirical tight-binding calculations yield $1.5,2.0,2.1$, and $1.7 \mathrm{eV}$, respectively. ${ }^{19,20}$ Although the reliability of such calculations for the unoccupied states and the absolute values of the band gaps is often questioned, the correspondence between experiment and calculation for $\bar{X}^{\prime}$ and $\bar{X}$ is quite good, whereas for $\bar{\Gamma}$ and particularly for $\bar{M}$ the theoretical values for the sur-

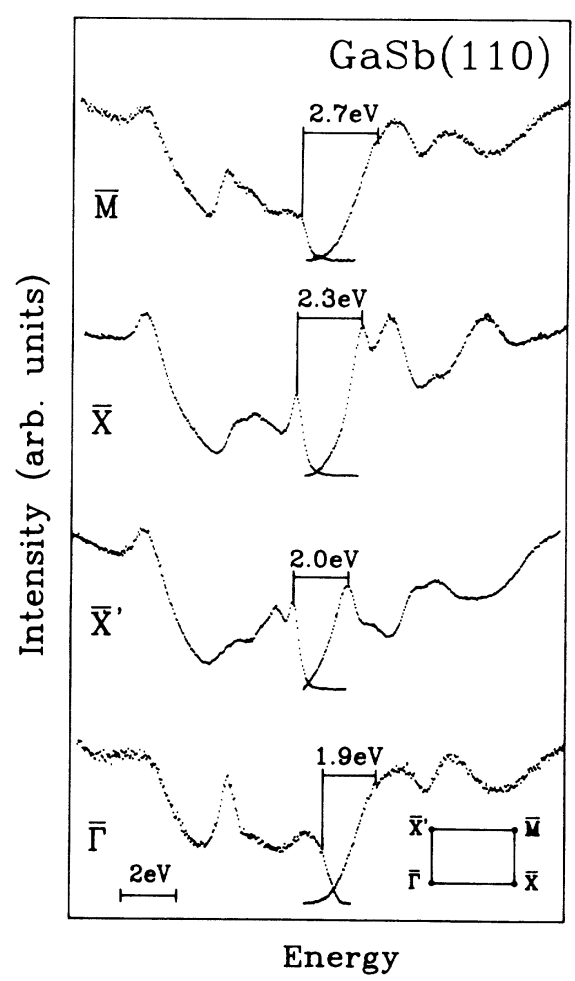

FIG. 3. Combined photoemission $(h v=21.2 \mathrm{eV})$ and inverse-photoemission $(h v=9.9 \mathrm{eV})$ spectra for the determination of surface band gaps of $\mathrm{GaSb}(110)$ at the high-symmetry points $\bar{\Gamma}, \bar{X}^{\prime}, \bar{X}$, and $\bar{M}$ of the surface Brillouin zone (inset). The electron angles in each spectrum are chosen so that the dangling-bond surface states (bars) are associated with kinetic energies corresponding to the symmetry points indicated. 
TABLE I. Surface band gaps (in eV) at the high-symmetry points for the six investigated III-V compound semiconductors. The fundamental bulk band gaps (in $\mathrm{eV}$ ) are also listed.

\begin{tabular}{llllll}
\hline \hline & $\bar{\Gamma}$ & $\bar{X}^{,}{ }^{\text {surf }}$ & $\bar{X}$ & $\bar{M}$ & $E_{\text {gulk }}^{\text {bul }}$ \\
\hline GaP & 3.0 & 3.5 & 3.7 & 3.6 & 2.26 \\
GaAs & 2.4 & 3.0 & 3.1 & 3.3 & 1.42 \\
GaSb & 1.9 & 2.0 & 2.3 & 2.7 & 0.72 \\
InP & 2.4 & 3.1 & 2.9 & 3.2 & 1.35 \\
InAs & 1.7 & 2.4 & 2.5 & 2.6 & 0.36 \\
InSb & 1.4 & 1.8 & 2.1 & 2.3 & 0.17 \\
\hline \hline
\end{tabular}

face gaps are much too small. Again, this trend is found for all studied semiconductors.

In the case of $\mathrm{GaAs}(110)$ a first ab initio calculation of the surface quasiparticle band structure has very recently been performed by Zhu et al. ${ }^{25}$ Our angle-resolved inverse-photoemission and photoemission data ${ }^{26}$ show a very similar dispersion and nearly the same energy position for the unoccupied DB surface band $C_{3}$, but a less satisfying correspondence for the occupied DB $A_{5}$. This is possibly the reason for the fact that the calculated surface gaps $\left(2.8 \mathrm{eV}\right.$ at $\bar{X}$ and around $3.0 \mathrm{eV}$ at $\bar{\Gamma}, \bar{X}^{\prime}$, and $\bar{M})$ do not compare so well with our experimental values $\left(2.4 \mathrm{eV}\right.$ at $\bar{\Gamma}, 3.0 \mathrm{eV}$ at $\bar{X}^{\prime}, 3.1 \mathrm{eV}$ at $\bar{X}$, and $3.3 \mathrm{eV}$ at $\left.\bar{M}\right)$. From a recent inverse-photoemission experiment of Perfetti and Reihl on $\operatorname{GaAs}(110),{ }^{7}$ the $A_{5}-C_{3}$ gap was deduced to be almost constantly $3.6 \mathrm{eV}$ over the entire Brillouin zone. This is a much larger value than the surface gaps measured in this study, and, moreover, pronounced variations of the surface gaps for the different highsymmetry points are observed in our experiments for all semiconductors, in strong disagreement with the results of Perfetti and Reihl. It should be realized that in their work the dispersion and energy position of the occupied dangling-bond state was not measured in situ, but was taken from the very early photoemission study of Huijser et al. ${ }^{3}$ By a similar procedure concerning the energy location of the occupied states, Straub et al. ${ }^{4}$ found for the smallest energy difference between the occupied and unoccupied DB surface states, $2.7 \mathrm{eV}$ at $\bar{\Gamma}$ and a 3-eVwide energy gap over a substantial part of the SBZ, which is in much better agreement with our experiments. The two very different results of Perfetti and Reihl ${ }^{7}$ and of Straub et al. ${ }^{4}$ show that the method of comparing ARIPES and ARPES results measured separately or even by different groups may cause errors because of serious difficulties in determining and adjusting the two energy scales accurately enough.

For a study of the chemical trend, Fig. 4 shows the combined photoemission and inverse-photoemission spectra at the $\bar{X}^{\prime}$ point of the surface Brillouin zone for all measured III-V compounds. All spectra show strong emission near their onset due to filled and empty dangling bonds, as expected for free surface states. The trend of increasing surface band gaps at $\bar{X}^{\prime}$ is the same as observed in the optical data ${ }^{12-14}$ (see below). The sequence for the other high-symmetry points (see Table I) is not exactly the same, but InSb has always the smallest and GaP the largest surface band gaps as for the bulk.

Figure 5 shows the measured surface band gaps at $\bar{\Gamma}$ (open circles) and $\bar{X}^{\prime}$ (solid circles), together with the low-lying optical transition energies measured by Cricenti et al. ${ }^{12,13}$ (triangles) and Berkovits et al. ${ }^{14}$ (squares) for all III-V compound semiconductors, plotted versus the fundamental bulk band gap. As seen from Fig. 5 and Table I the smallest surface band gap is that at $\bar{\Gamma}$, and it depends approximately linearly but at least monotonically on the bulk gap; however, its absolute values are about $1 \mathrm{eV}$ larger. For $\bar{X}^{\prime}$ the relation is not so simple as seen, for instance, by the anomaly for InAs and $\mathrm{GaSb}$ in the $E_{g}^{\text {surf }}-E_{g}^{\text {bulk }}$ plot, and also for InP and GaAs, but not so distinct. Taken by themselves, the $\mathrm{Ga}$ and In compounds again show a monotonic behavior. It should be noted here that the surface $\bar{X}^{\prime}$ point lies in the middle between the $\Gamma$ and $X$ points of the projected bulk Brillouin zone, and band-structure calculations for InAs and $\mathrm{GaSb}$ of Chelikowsky and Cohen ${ }^{27}$ predict a change in the sequence of the bulk gaps from $\Gamma$ to $X$. Therefore, it seems

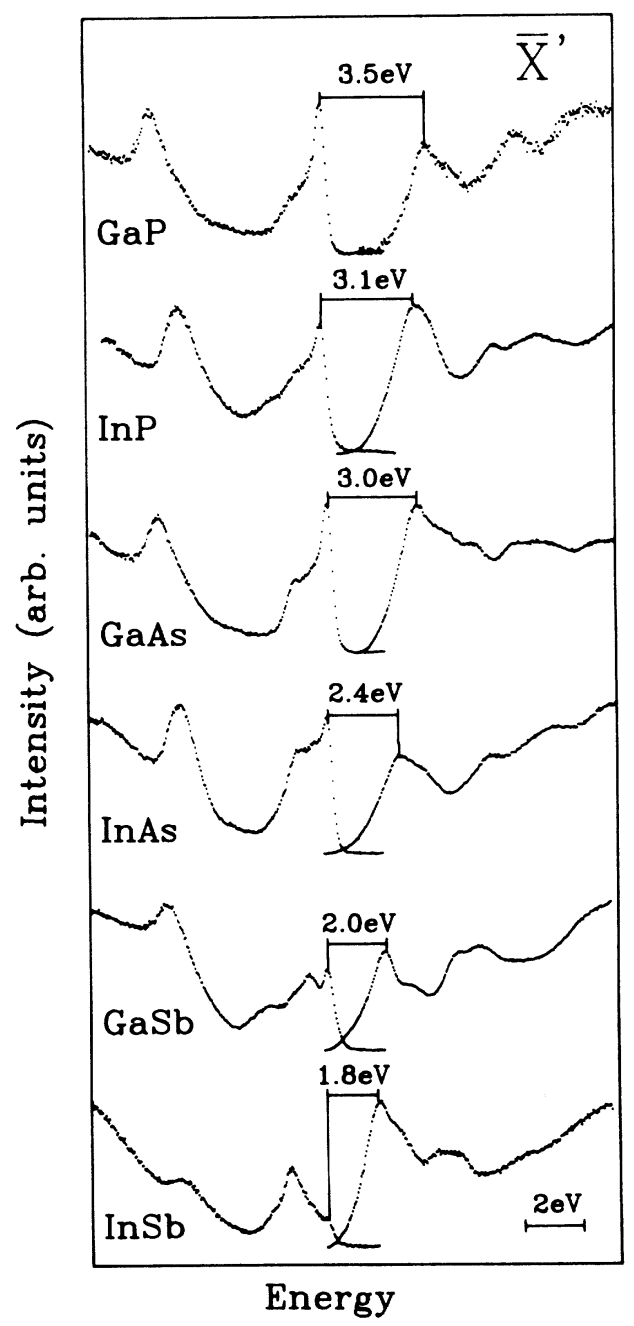

FIG. 4. Photoemission and inverse-photoemission spectra at the $\bar{X}^{\prime}$ point for the (110) cleavage planes of six III-V semiconductor compounds ordered according to increasing surface band gaps. 


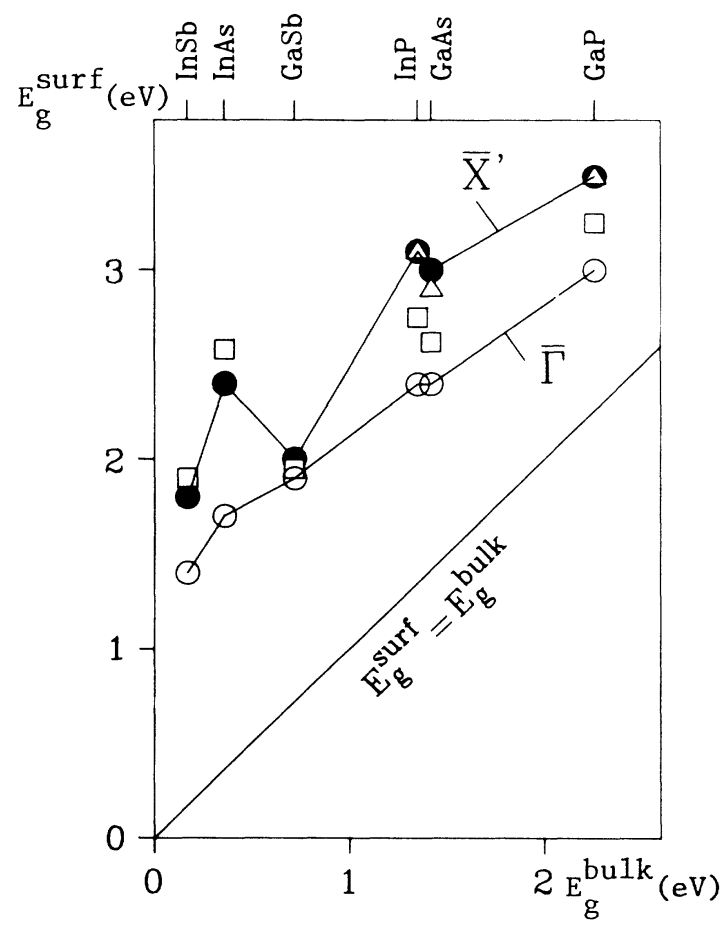

FIG. 5. Surface band gaps at points $\bar{\Gamma}$ (open circles) and $\bar{X}^{\prime}$ (solid circles) and the low-lying surface-sensitive optical transition energies measured by Cricenti et al. (Refs. 12 and 13) (triangles) and Berkovits et al. (Ref. 14) (squares) of six III-V semiconductor compounds plotted against their bulk band gaps.

that there is a correlation between the surface and the bulk gap also for $\bar{X}^{\prime}$. It should be noted that the surface band gaps for $\operatorname{InP}(110)$ and $\mathrm{GaAs}(110)$ are very similar, as suggested from the nearly equal bulk band gaps. We have also compared the surface band gaps at $\bar{\Gamma}$ and $\bar{X}^{\prime}$ with the indirect but more $p$-like $\Gamma_{8}^{v}-X_{6}^{c}$ bulk band gap. Although we do not achieve a better correspondence for all six semiconductors, a reasonable correlation is found for the Ga- and In-containing compounds separately.

\section{COMPARISON WITH OPTICAL AND ELECTRON-ENERGY-LOSS EXPERIMENTS}

As seen in Fig. 3 and discussed above, the danglingbond states show a pronounced emission at the $\bar{X}^{\prime}$ and $\bar{X}$ points, whereas at points $\bar{\Gamma}$ and $\bar{M}$ the peaks are only weak. Neglecting matrix elements and taking the peak intensities of photoemission and inverse photoemission as a rough measure for the momentum-resolved density of states, we would expect that photon- or electron-induced transitions between the filled and empty dangling-bond states predominantly take place at the zone boundary near $\bar{X}^{\prime}$ and $\bar{X}$. With the polarization-modulatedreflectivity (PMR) method, Berkovits et al. ${ }^{14}$ have studied the (110) surfaces of the six III-V compound semiconductors. They detect three surface-sensitive optical transitions, labeled $S_{1}, S_{2}$, and $S_{3}$, excited by the light polarized along the [110] direction. The $S_{1}$ transition energies (squares in Fig. 5) are very close to the surface band gaps at the $\bar{X}^{\prime}$ point for the narrow-gap semiconductors and about $300 \mathrm{meV}$ smaller for InP, GaAs, and GaP. The anomaly in the sequence for InAs and $\mathrm{GaSb}$ as well as for InP and GaAs are also clearly observed in the optical spectra for $S_{1}$. The next optical transitions $\left(S_{2}\right)$ lie about $200 \mathrm{meV}$ higher in energy and are interpreted as transitions between the filled DB state $\left(A_{5}\right)$ and the empty back bond state $\left(C_{4}\right)$. However, considering our photoemission data an assignment of the excitation $S_{2}$ to transitions between $A_{5}$ and $C_{3}$ at a different high-symmetry point (here the $\bar{X}$ point) seems to be also possible. The $S_{3}$ transitions occur for all crystals at higher energies then the values we found for the surface band gaps between $A_{5}$ and $C_{3}$, so other surface states must be involved.

Cricenti et al. have investigated the complex dielectric function for the (110) surfaces of $\mathrm{GaAs}, \mathrm{GaP},{ }^{12}$ and InP, ${ }^{13}$ using polarized and unpolarized light by surfacedifferential-reflectivity (SDR) spectroscopy. The unpolarized optical transitions taken from the maxima of the imaginary part of the surface dielectric function (triangles in Fig. 5) are about $300 \mathrm{meV}$ higher in energy than the first optical transition observed by Berkovits et al. ${ }^{14}$ and have the same energy within experimental accuracy as the surface band gaps at the $\bar{X}^{\prime}$ point. For GaAs(110) and $\mathrm{GaP}(110)$ additional polarized optical transitions are observed at 3.5 and $2.8 \mathrm{eV}$, respectively, and for $\operatorname{InP}(110)$ two unpolarized optical transitions with higher energies (3.6 and $3.8 \mathrm{eV}$ ) are found (not shown in Fig. 5). The 3.5-eV emission for GaAs and the 3.6- and 3.8-eV emissions for InP are distinctly larger than the observed surface band gaps between the dangling-bond states and point to transitions involving other surface states. The 2.8-eV transition for GaP, also observed by Berkovitz et al. ${ }^{14}$ is $200 \mathrm{meV}$ smaller than the surface band gap at $\bar{\Gamma}$ and, thus, could only be explained by surface excitonic effects or by indirect transitions between the DB surface states.

Studying the azimuthal dependence of the electronic excitations by high-resolution electron-energy-loss spectroscopy (HREELS), Nannarone et al. ${ }^{10}$ found surfacesensitive broad structures with a maximum at $2.9 \mathrm{eV}$ for $\mathrm{GaAs}(110)$ and at 3.5 and $5.6 \mathrm{eV}$ for $\mathrm{GaP}(110)$. The lowlying transitions correspond very well with the surface band gaps at the $\bar{X}^{\prime}$ point $(3.0 \mathrm{eV}$ for $\mathrm{GaAs}$ and $3.5 \mathrm{eV}$ for GaP). With the same techniques, Del Pennino et al. ${ }^{11}$ observed a series of energy losses between 1 and 7 $\mathrm{eV}$ with different surface and bulk character for GaAs(110). They assign a weak structure at $2.6 \mathrm{eV}$ to a transition between the filled As and empty Ga dangling bond, in reasonable correspondence with our surface band gap at the $\bar{\Gamma}$ point $(2.4 \mathrm{eV})$. A sharp structure at 3 $\mathrm{eV}$ was attributed to a mainly bulklike transition, although the authors cannot exclude the superposition of surface excitations. Upon comparison with our data, we would assign this to transitions between the DB surface states near the $\bar{X}^{\prime}$ or $\bar{X}$ point. Summarizing the comparison with the optical and HREELS data, we find quite a good correspondence between our directly determined surface band gaps for $\bar{X}^{\prime}$ and $\bar{X}$ and the low-lying surface transitions. 


\section{CONCLUSIONS}

The in situ combination of angle-resolved photoemission and inverse photoemission without use of any additional reference level like the Fermi level or the valenceband maximum is a powerful tool to determine directly the energy difference of occupied and unoccupied surface states at different points in the Brillouin zone. The results for the (110) surface of III-V compound semiconductors show a correlation with the bulk band gap. At the zone boundary the gaps correspond well with the low-lying transitions observed in optical and electronenergy-loss spectroscopies. The experimental values of the surface band gaps at high-symmetry points can be compared directly with theory and should serve as cru- cial parameters to check the reliability of existing and future band-structure calculations. However, for a better understanding of all experimental data, more elaborate calculations of the surface band structure including many-body effects, particularly for the unoccupied bands, are still necessary and of fundamental interest.

\section{ACKNOWLEDGMENTS}

We thank J. Henk (Universität Kiel) for discussions on theoretical aspects. This work was supported by the Deutsche Forschungsgemeinschaft (Bonn, Germany) under Project No. SK-13/4, and in part by the Bundesministerium für Forschung und Technologie (Bonn, Germany), under Project No. 05-301-AAI.
${ }^{1}$ G. V. Hansson and R. I. G. Uhrberg, Surf. Sci. Rep. 9, 197 (1988), and references therein.

${ }^{2}$ N. V. Smith, Rep. Prog. Phys. 51, 1227 (1988), and references therein.

${ }^{3}$ A. Huijser, J. van Laar, and T. L. van Rooy, Phys. Lett. 65A, 337 (1978).

${ }^{4}$ D. Straub, M. Skibowski, and F. J. Himpsel, Phys. Rev. B 32, 5237 (1985).

${ }^{5}$ D. Straub, M. Skibowski, and F. J. Himpsel, J. Vac. Sci. Technol. A 3, 1484 (1985).

${ }^{6}$ W. Drube, D. Straub, and F. J. Himpsel, Phys. Rev. B 35, 5563 (1987).

${ }^{7}$ P. Perfetti and B. Reihl, Phys. Scr. T25, 173 (1989).

${ }^{8}$ S. Selci, F. Ciccacci, G. Chiarotti, P. Chiaradia, and A. Cricenti, J. Vac. Sci. Technol. A 5, 327 (1987).

${ }^{9}$ V. L. Berkovits, V. A. Kiselev, and V. I. Safarov, Surf. Sci. 211/212, 489 (1989).

${ }^{10}$ S. Nannarone, S. D'Addato, J. A. Schaefer, Y. Chen, J. Anderson, and G. J. Lapeyre, Surf. Sci. 211/212, 524 (1989).

${ }^{11}$ U. Del Pennino, M. G. Betti, C. Mariani, and I. Abbati, Surf. Sci. 207, 133 (1988).

${ }^{12}$ A. Cricenti, S. Selci, F. Ciccacci, A. C. Felici, C. Goletti, Zhu Yong and G. Chiarotti, Phys. Scr. 38, 199 (1988).

${ }^{13}$ A. Cricenti, S. Selci, A. C. Felici, C. Goletti, and G. Chiarotti, Surf. Sci. 211/212, 552 (1989).

${ }^{14}$ V. L. Berkovits, L. F. Ivantsov, I. V. Makarenko, T. A. Minashvili, and V. I. Safarov, Solid State Commun. 64, 767 (1987).

${ }^{15}$ D. J. Chadi, Phys. Rev. B 18, 1800 (1979); J. Pollmann, in Festkörperprobleme (Advances in Solid State Physics), edited by P. Grosse (Pergamon/Viewig, Braunschweig, FRG, 1980), Vol. 20, p. 117, and references therein.

${ }^{16}$ A. U. MacRae and G. W. Gobeli, J. Appl. Phys. 35, 1629 (1964).

${ }^{17}$ C. Mailhiot, C. B. Duke, and D. J. Chadi, Surf. Sci. 149, 366 (1985).

${ }^{18}$ S. Y. Tong, W. N. Mei, and G. Xu, J. Vac. Sci. Technol. B 2, 393 (1984).

${ }^{19}$ R. P. Beres, R. E. Allen, and J. D. Dow, Phys. Rev. B 26, 5702 (1982); Solid State Commun. 45, 13 (1983).

${ }^{20}$ C. Mailhiot, C. B. Duke, and D. J. Chadi, Phys. Rev. B 31, 2213 (1985).

${ }^{21}$ N. Babbe, W. Drube, I. Schäfer, and M. Skilbowski, J. Phys. E 18, 158 (1985).

${ }^{22}$ I. Schäfer, W. Drube, M. Schlüter, G. Plagemann, and M. Skibowski, Rev. Sci. Instrum. 58, 710 (1987).

${ }^{23}$ H. Carstensen, R. Manzke, I. Schäfer, and M. Skibowski, in The Physics of Semiconductors, edited by O. Engström (World Scientific, Singapore, 1987), Vol. 1, p. 125; R. Manzke, H.-P. Barnscheidt, C. Janowitz, and M. Skibowski, Phys. Rev. Lett. 58, 610 (1987).

${ }^{24}$ S. M. Sze, Physics of Semiconductor Devices (Wiley, New York, 1981), and references therein.

${ }^{25}$ X. Zhu, S. B. Zhang, S. G. Louie, and M. L. Cohen, Phys. Rev. Lett. 63, 2112 (1989).

${ }^{26}$ H. Carstensen, R. Claessen, R. Manzke, and M. Skibowski (unpublished).

${ }^{27}$ J. R. Chelikowsky and M. L. Cohen, Phys. Rev. B 14, 556 (1976). 